\title{
AFFINE ENDOMORPHISMS WITH A DENSE ORBIT
}

\author{
TATSURO KASUGA
}

\begin{abstract}
ABSTRACr. For a continuous endomorphism $f$ on a locally compact group $X$ and $a \in X$, we define an affine endomorphism $f_{a}: X \rightarrow X$. We prove that if $f_{a}$ is not one to one and if $\left(X, f_{a}\right)$ has a dense orbit then $X$ is compact.
\end{abstract}

Let $X$ be a locally compact group and let $f_{a}: X \rightarrow X$ be an affine endomorphism: i.e. $f_{a}(x)=a f(x)$ where $a$ is a fixed element of $X$ and $f$ is a continuous endomorphism of $X$ onto itself. The following problem is raised by P. R. Halmos [4, p. 29]. "Can an automorphism of a locally compact but noncompact group be an ergodic measure-preserving transformation?" Recently the problem of Halmos was solved by N. Aoki [1]. Halmos' problem was generalized by Dani and Dateyama-Kasuga who obtained the following:

THEOREM A. Let $X$ be a locally compact metric group and $f_{a}: X \rightarrow X$ be a bicontinuous affine automorphism. If $\left(X, f_{a}\right)$ has a dense orbit, then

(i) $X$ is compact when $X$ is connected ( $S$. G. Dani [2]).

(ii) $X$ is either compact or discrete when $X$ is totally disconnected [3].

The aim of this note is to investigate whether the problem holds for continuous affine endomorphisms. The following is the main result of this note.

THEOREM 1. Let $X$ be a locally compact group and let $f_{a}: X \rightarrow X$ be a continuous affine endomorphism that is not one-to-one. If $\left(X, f_{a}\right)$ has a dense orbit then $X$ is compact.

We will deduce Theorem 1 from the following consequence of Theorem A.

THEOREM B. Let $X$ be a locally compact metric group and $f_{a}: X \rightarrow X$ be a bicontinuous affine automorphism. If $X$ is not discrete and $\left(X, f_{a}\right)$ has a dense orbit, then $X$ is compact.

Proof. Let $X_{0}$ be the connected component of the identity $e$ in $X$. Then $X / X_{0}$ is a locally compact totally disconnected metric group. Now define the map $g_{a}: X / X_{0} \rightarrow$ $X / X_{0}$ by

$$
g_{a}\left(x X_{0}\right)=f_{a}(x) X_{0} \quad(x \in X) .
$$

Then it is obvious that $g_{a}$ is a bicontinuous affine automorphism and $\left(X / X_{0}, g_{a}\right)$ has a dense orbit. By Theorem A, $X / X_{0}$ is compact. Therefore it is enough to show that $X_{0}$ is compact. This is proved by using the fact that an affine automorphism

Received by the editors July 11, 1984 and, in revised form, February 15, 1985.

1980 Mathematics Subject Classification. Primary 54H20; Secondary 22D05. 
with a dense orbit is measure-preserving under the left Haar measure $\mu$ (cf. [3, Proposition 1]).

The conclusion of Theorem 1 will be obtained in proving the following two propositions:

Proposition 2. Let $X$ and $f_{a}$ be as in Theorem 1. If $X$ is discrete and $\left(X, f_{a}\right)$ has a dense orbit, then $X$ is finite.

Proof. Since $f_{a}$ is not one-to-one, there are $z_{1}$ and $z_{2}$ in $X$ such that $z_{1} \neq z_{2}$, but $f_{a}\left(z_{1}\right)=f_{a}\left(z_{2}\right)$. Since $\left(X, f_{a}\right)$ has a dense orbit, there is an element $x_{0}$ in $X$ such that $\left\{f_{a}^{n}\left(x_{0}\right): n \geqslant 0\right\}$ is dense in $X$. Hence there are integers $n_{1}$ and $n_{2}\left(0<n_{1}<n_{2}\right)$ such that $f_{a}^{n_{1}}\left(x_{0}\right)=z_{1}$ and $f_{a}^{n_{2}}\left(x_{0}\right)=z_{2}$. Since $f_{a}^{n_{1}+1}\left(x_{0}\right)=f_{a l}\left(z_{1}\right)=f_{a}\left(z_{2}\right)=$ $f_{a}^{n_{2}+1}\left(x_{0}\right)$, we have

$$
X=\left\{x_{0}, f_{a}\left(x_{0}\right), f_{a}^{2}\left(x_{0}\right), \ldots, f_{a}^{n_{2}-n_{1}-1}\left(x_{0}\right)\right\},
$$

i.e. $X$ is finite.

For the case $X$ is not discrete, $X$ is $\sigma$-compact when $\left(X, f_{a}\right)$ has a dense orbit. For, since $X$ is locally compact, there exists a compact neighborhood $V$ of $e$ in $X$. By assumption, there is $x_{0} \in X$ such that $\left\{f_{a}^{n}\left(x_{0}\right): n \geqslant 0\right\}$ is dense in $X$. Hence, $X=\cup_{n=0}^{\infty} f_{a}^{n}\left(x_{0}\right) V$, i.e. $X$ is $\sigma$-compact. Clearly, $f_{a}$ is an open map, because $f: X \rightarrow X$ is onto.

Hereafter we may assume that $X$ is $\sigma$-compact. Then there exists a compact normal subgroup $H$ of $X$ such that $f(H) \subset H$ and that $X / H$ is metrizable and separable (see [1]). Define the endomorphism $g: X / H \rightarrow X / H$ by $g(x H)=f(x) H$ and put $g_{a}(x H)=a g(x H)(x \in X)$. If $\left(X, f_{a}\right)$ has a dense orbit, then so does $\left(X / H, g_{a}\right)$.

PROPOSITION 3. Let $X$ be a locally compact metric group with a left invariant metric $d$ and let $f_{a}: X \rightarrow X$ be a continuous affine endomorphism. If $X$ is not discrete and if $\left(X, f_{a}\right)$ has a dense orbit, then $X$ is compact.

Proof. Define a metric $d_{0}$ by

$$
d_{0}(x, y)=\frac{d(x, y)}{d(x, y)+1} \quad(x, y \in X) .
$$

Then $d_{0}$ is bounded and is equivalent to $d$. We let

$$
\bar{X}=\left\{\left(x_{i}\right)_{i=0}^{\infty}: f\left(x_{i+1}\right)=x_{i}, i \geqslant 0\right\} .
$$

Put $\bar{f}\left(\left(x_{i}\right)_{i=0}^{\infty}\right)=\left(f\left(x_{i}\right)\right)_{i=0}^{\infty}$ and $\varphi_{0}\left(\left(x_{i}\right)_{i=0}^{\infty}\right)=x_{0}$ for $\left(x_{i}\right)_{i=0}^{\infty} \in \bar{X}$. Then $\bar{f}$ is a continuous group automorphism and $\varphi_{0} \circ \bar{f}=f \circ \varphi_{0}$ holds. Define the metric for $\bar{X}$ by

$$
\bar{d}(\bar{x}, \bar{y})=\sum_{i=0}^{\infty} d_{0}\left(x_{i}, y_{i}\right) / 2^{i} \quad\left(\bar{x}=\left(x_{i}\right)_{i=0}^{\infty}, \bar{y}=\left(y_{i}\right)_{i=0}^{\infty} \in \bar{X}\right) .
$$

Clearly $\bar{X}$ is a locally compact metric group and $\bar{d}$ is rotation invariant since $d_{0}$ is rotation invariant. For $\bar{a}=\left(a_{i}\right)_{i=0}^{\infty} \in \bar{X}$ with $a_{0}=a$, and affine endomorphism $\bar{f}_{\bar{a}}$ has a dense orbit in $\bar{X}$ since so does $\left(X, f_{a}\right)$. Indeed, we can find $z_{0} \in X$ such that 
$\left\{f_{a}^{n}\left(z_{0}\right) ; n \geqslant 0\right\}$ is dense in $X$. We fix the point $z_{0}$. Since $f$ is onto, there is $\bar{y}=\left(y_{i}\right)_{i=0}^{\infty} \in \bar{X}$ with $y_{0}=z_{0}$ such that $f\left(y_{i+1}\right)=y_{i}$ for $i \geqslant 0$. Let $\varepsilon>0$. For $\bar{x}=\left(x_{i}\right)_{i=0}^{\infty} \in \bar{X}$, there is $m>0$ such that

$$
\sum_{i=m+1}^{\infty} d_{0}\left(x_{i}, y_{i}\right) / 2^{i}<\varepsilon / 2
$$

and so we choose $\eta>0$ such that $d_{0}\left(f^{j}(x), f^{j}(y)\right)<\varepsilon / 4 \quad(0 \leqslant j \leqslant m)$ when $d_{0}(x, y)<\eta$. Take $\bar{a}=\left(a_{i}\right)_{i=0}^{\infty} \in \bar{X}$ such that $a_{0}=a$ and $f^{j}\left(a_{i}\right)=a_{j-i}(0 \leqslant i \leqslant j$ $\leqslant m)$. Since $\left\{f_{a}^{n}\left(z_{0}\right) ; n \geqslant 0\right\}$ is dense in $X$, for $a_{1}^{-1} a_{2}^{-1} \cdots a_{m}^{-1} x_{m} \in X$, there is $k>0$ such that

$$
d_{0}\left(f_{a}^{k}\left(z_{0}\right), a_{1}^{-1} a_{2}^{-1} \cdots a_{m}^{-1} x_{m}\right)<\eta
$$

Hence

$$
\begin{aligned}
& d_{0}\left(a_{m-j} a_{m-j-1} \cdots a_{2} a_{1} f_{a}^{k+j}\left(z_{0}\right), f^{j}\left(x_{m}\right)\right) \\
& \quad=d_{0}\left(a_{m-j} a_{m-j-1} \cdots a_{2} a_{1} a_{0} f\left(a_{0}\right) \cdots f^{j-1}\left(a_{0}\right) f^{j}\left(f_{a}^{k}\left(z_{0}\right)\right), f^{j}\left(x_{m}\right)\right) \\
& \quad=d_{0}\left(f^{j}\left(f_{a}^{k}\left(z_{0}\right)\right), f^{j}\left(a_{1}^{-1}\right) f^{j}\left(a_{2}^{-1}\right) \cdots f^{j}\left(a_{m}^{-1}\right) f^{j}\left(x_{m}\right)\right)<\varepsilon / 4
\end{aligned}
$$

for $0 \leqslant j \leqslant m$. Therefore,

$$
\begin{aligned}
\bar{d}\left(\bar{f}_{\bar{a}}^{k+m-1}(\bar{y}), \bar{x}\right) & \leqslant \sum_{j=0}^{m} d_{0}\left(a_{m-j} a_{m-j-1} \cdots a_{2} a_{1} f_{a}^{k+j}\left(z_{0}\right), f^{j}\left(x_{m}\right)\right) / 2^{j}+\varepsilon / 2 \\
& <\varepsilon,
\end{aligned}
$$

which implies that $\left(\bar{X}, \bar{f}_{\bar{a}}\right)$ has a dense orbit.

Obviously $\bar{X}$ is locally compact and $\bar{f}_{\bar{a}}: \bar{X} \rightarrow \bar{X}$ is one-to-one. Since $\bar{f}_{\bar{a}}$ is an open map, actually $\bar{f}_{\bar{a}}$ is bicontinuous. Since $\left(\bar{X}, \bar{f}_{\bar{a}}\right)$ has a dense orbit and $\bar{X}$ is not discrete, $\bar{X}$ is compact by Theorem B. Hence $\varphi_{0}(\bar{X})=X$ is compact. The proof is completed.

\section{REFERENCES}

1. N. Aoki, Dense orbits of automorphisms and compactness of groups, Topology Appl. (to appear).

2. S. G. Dani, Dense orbits of affine automorphisms and compactness of groups, J. London Math. Soc. (2) 25 (1982), 241-245.

3. M. Dateyama and T. Kasuga, Ergodic affine maps of locally compact groups, J. Math. Soc. Japan (1985), 363-372.

4. P. R. Halmos, Lectures on ergodic theory, Publ. Math. Soc. Japan, Tokyo, 1956.

5. D. Montgomery and L. Zippin, Topological transformation groups, Interscience, New York, 1955.

Department of Mathematics, Kumamoto Radio Technical College, Nishigoshi-Machi, KIKUCHI-GUN, KUMAMOTO, 861-11, JAPAN 